\author{
E-ISSN: 2321-9637 \\ Available online at www.ijrat.org
}

\title{
Web Software Engineering: Principles, Trends, Future Challenges
}

\author{
Manas Kumar Yogi ${ }^{1}$, K.Chandrasekhar, \\ Asst. Prof. CSE Dept. ${ }^{2,}$, Pragati Engineering College(A),Surampalem \\ Email: manas.yogi@gmail.com ${ }^{1}$,sekhar222.k@gmail.com ${ }^{2}$
}

\begin{abstract}
Inside a brief period, the Internet and World Wide Web have turned out to be universal, outperforming all other mechanical improvements in our history. They've likewise developed quickly in their extension and degree of utilization, altogether influencing all parts of our lives. Businesses, for example, assembling, travel and cordiality, banking, instruction, and government are Web-empowered to improve and upgrade their activities. Web based business has extended rapidly, cutting crosswise over national limits. Indeed, even conventional inheritance data and database frameworks have moved to the Web. Advances in remote innovations and Webempowered machines are setting off another rush of versatile Web applications. Accordingly, we progressively rely upon a scope of Web applications. Since a significant number of us depend on Web based frameworks and applications, they should be solid and perform well. To manufacture these frameworks and applications, Web designers need a sound system, a trained and repeatable procedure, better improvement instruments, and a lot of good rules. The rising field of Web building satisfies these necessities. It utilizes logical, designing, and the board standards and methodical ways to deal with effectively create, send, and keep up excellent Web frameworks and applications. It intends to acquire the present turmoil Web based framework advancement leveled out, limit dangers, and upgrade Web webpage viability and quality.
\end{abstract}

Index Terms-Web, Software Engineering, Personalization, Retrieval, Efficiency

\section{INTRODUCTION}

The development of the Internet, Intranets, Extranets, and the World Wide Web has just significantly affected business, trade, industry, saving money and back, training, government and amusement areas, and our individual and working life. Numerous heritage data and database frameworks are being moved to the Internet and the Web situations. Electronic business through the Internet is quickly developing, cutting crosswise over national limits. An extensive variety of new, complex appropriated applications is rising in the Web condition. The prevalence and omnipresence originates from the idea of the Web itself and its highlights: it gives a data portrayal that backings interlinking of a wide range of substance, simple access for end clients, and simple substance creation utilizing generally accessible devices. In any case, by and large, the improvement approach utilized for Web-based frameworks has been impromptu, and Web-based frameworks have been continued going through a persistent stream of patches. Generally speaking, Web-based framework improvement needs meticulousness, a methodical methodology, and quality control and confirmation. As the many-sided quality and refinement of Electronic applications develop, there is currently real and developing worry about the way in which they are made and their long haul quality and uprightness.
Without restrained process for creating Web-based frameworks, we may confront difficult issues in their fruitful advancement, sending, activity of and 'upkeep.' Poorly created Web-based applications that are mushrooming now have a high likelihood of disappointment. More awful, as Web-based frameworks develop more intricate, a disappointment in one can and will proliferate expansive based issues crosswise over many. At the point when this occurs, trust in the Web might be shaken hopelessly, causing a Web emergency. The potential Web emergency could be more genuine and across the board than the product emergency, which the product designers have been facing. In request to maintain a strategic distance from a conceivable Web emergency and make more prominent progress being developed and uses of complex Web-based frameworks, there is a squeezing requirement for trained methodologies and new strategies and devices for advancement, arrangement and assessment of Web-based frameworks. Critically, such methodologies and systems must consider 1) the exceptional highlights of the new medium, 2) the operational conditions, and 3) situations and variety of client profiles, and in addition 4) the sort (and aptitudes and information) of the general population building Web-based frameworks. These represent extra difficulties to Web-based application development. 
International Journal of Research in Advent Technology, Vol.7, No.5, May 2019

\section{E-ISSN: 2321-9637 \\ Available online at www.ijrat.org}

This paper endeavors to survey the issues of Webbased framework improvement as is presently polished in numerous cases and contends the requirement for embracing Web Engineering approaches for creating adaptable, quality, vast scale Web-based frameworks. It introduces the standards and jobs of Web Engineering and surveys the likenesses and contrasts between advancement of customary programming and Web-based frameworks, and between programming designing and Web building. It distinguishes key Web building exercises and layouts methodologies and strategies for orderly advancement of Web-based applications checking on continuous work here. It likewise examines the possibilities of Web building and features the regions that need additionally study and advancement.

Numerous properties of value Web-based Systems, for example, simplicity of route, availability, versatility, maintainability, usability, similarity and interoperability, security, lucidness, and dependability are not given due thought amid advancement. Numerous designers appear to be ignorant of the main problems and difficulties confronting significant Webbased application improvement and its consistent support. There is a need to induce a familiarity with the requirement for more trained ways to deal with Web-based application improvement, and we have to move from the current, to a great extent specially appointed (and customized) way to deal with a superior restrained methodology and process. Likewise we have to understand that Web-based framework improvement isn't simply visual depiction or substance advancement any more, and there are developing number of complex applications - intranetbased applications, value-based frameworks, and other e-business applications. "There is a whole other world to Web webpage than visual outline and UI. Sites are ending up more like projects less like static archives", and thus Web-based frameworks improvements are ending up more like programming ventures, and less like show-stopper. There is real and developing worry about the impromptu way in which most Web-based frameworks are right now made and their long haul quality and honesty. More modernity and high manysided quality of new Web-based applications get numerous new difficulties that should be acceptably tended to.

\section{Need for web software engineering:}

Three of the effective quality factors for success of web applications are as below:

\section{Reliability}

2. Usability

3. Security

In addition these four indispensible criteria are:

4. Availability

5. Scalability

6. Maintainability

7. Time-to-market

1. Reliability: This is an extremely natural quality trait that is related with a broad research literature and a gathering of business instruments for testing, guaranteeing, guaranteeing, and estimating the dependability of programming. The need of exceedingly dependable programming is additionally natural in programming applications that are security basic, for example, media communications, aviation, and therapeutic gadgets. Albeit a large number of us who are specialists in these territories are hesitant to let it be known, in all actuality the majority of the product that is as of now delivered does not should be exceptionally solid. The portions of the business specified above are moderately small. Web programming, be that as it may, is basic to the business achievement of numerous organizations and if the product does not work dependably, the organizations won't succeed. The client base for web programming is vast and they come anticipating that the web applications should fill in as dependably on the off chance that they are heading off to the market or calling to arrange from an index. In addition, if a web application does not function admirably, the clients don't need to drive further to achieve another store, they basically need to indicate their program an alternate URL. In this manner, if web programming is problematic, sites that rely upon the product will lose clients and the organizations may lose huge amount of cash. These elements join to make unwavering quality of web programming essential, and above all, organizations can stand to spend assets to guarantee high dependability. To be sure, they can't bear the cost of not to.

2. Usability: As said above, most web programming applications have a wide client base. These clients have developed to anticipate that product will be extremely easy to figure out how to use, as straightforward as purchasing an item at a store. In spite of the fact that a ton of information is accessible for how to create usable programming and web sites, many sites still don't meet the ease of use necessities that a large portion of us anticipate. This, combined 
International Journal of Research in Advent Technology, Vol.7, No.5, May 2019

\section{E-ISSN: 2321-9637 \\ Available online at www.ijrat.org}

with the way that clients display little webpage steadfastness, implies that sites that are not usable won't be utilized: clients will rapidly change to more usable sites when they are put on the web.

3. Security: We have all caught wind of the ongoing situations where sites have been broken into and private client data circulated or held for payoff. This is just a single case of the numerous potential security issues in web programming applications. At the point when the primary utilization of the web was to appropriate online pamphlets, the results of security breaks were generally little. With the substantially more extensive uses today, nonetheless, organization's sites that are broken into face noteworthy misfortunes in income, expansive repair costs, legitimate outcomes and can lose validity with their clients. In this way, it is basic that web programming applications handle client information and other electronic data as safely as would be prudent. One of the quickest developing exploration regions in software engineering is that of programming security, and web programming designers are confronting a gigantic deficit both as far as accessible learning and work force who have the information that is accessible.

4. Availability: On the web, clients not just expect accessibility "day in and day out", they anticipate that the site will be operational each day of the year - "all day, every day/365". Indeed, even a ten moment down-time can be damaging. Availability implies something other than being up and running day in and day out/365, accessibility additionally implies that the web programming must be accessible when gotten to by differing programs. The apparently endless program wars of the previous couple of years have implied that product sellers effectively endeavor to ensure that their product won't work under aggressive programs. By utilizing highlights that are accessible for one program or on one stage, web programming engineers progress toward becoming "infantrymen" in the program wars, now and then accidentally. As an precedent, many noteworthy sites utilize "shockwaveflash", which implies that the numerous clients of Unix and Netscape can't see their own site. To be accessible in this sense, sites must adjust their introductions to work with all programs, which requires essentially more information and exertion with respect to the designers.

5. Scalability: web programming applications must be set up to become rapidly both regarding number of clients adjusted and as far as administrations advertised. The requirement for adaptability has been a driver for a significant part of the innovation advancements of the previous couple of years. The business has grown new programming dialects, new plan techniques, and new correspondence and information exchange conventions, to some extent to permit sites to develop as required. Adaptability additionally straightforwardly impacts different characteristics. A cliché that any programming instructor knows is that any plan will work for little classroom works out, however extensive programming applications require control and inventiveness. As sites develop, little shortcomings in the product that did not cause issues in activity can prompt disappointments (unwavering quality issues), ease of use issues, and security breaks. Planning and building web programming applications that can be effortlessly scaled is as of now a standout amongst the most fascinating and critical difficulties in programming outline.

6. Maintainability: One novel part of web programming frameworks is the recurrence of new releases, or then again the refresh rate. Introducing customary programming includes promoting, deals and sending or even close to home establishment at clients' destinations. Since this procedure is extremely costly, expansive quantities of upkeep adjustments are generally gathered after some time and appropriated to clients in the meantime. On the off chance that a product item is discharged today, the engineers will begin building up a rundown of fundamental changes. On the off chance that the main change is basic (say, changing the mark on a catch), the alteration might be made instantly. Yet, the postponement in discharges implies that adjustment won't be accessible to the clients for quite a long time, if not years. With web programming, notwithstanding, the refresh rate is substantially quicker. Upkeep updates can be introduced and quickly made accessible to clients through the site. Accordingly, even little individual changes, (for example, changing the mark on a catch) can be introduced instantly. One consequence of this is of upkeep cycles of months or years, sites can have support cycles of days or even hours. Albeit past programming applications have had high upkeep prerequisites, and there has even been investigation into alleged "on the fly" support for specific applications, incessant and consistent upkeep refreshes have at no other time been fundamental for much the business programming. Another repercussion of 
International Journal of Research in Advent Technology, Vol.7, No.5, May 2019

\section{E-ISSN: 2321-9637 \\ Available online at www.ijrat.org}

expanded refresh rate needs to do with similarity. Clients don't generally redesign their product, in this way programming sellers must guarantee similarity of new forms with old adaptations. Organizations can control the appropriation of web programming to take out that kind of similarity. Obviously, this is supplanted by program similarity; web applications must have the capacity to run effectively on a few internet browsers, and various forms of every program. One probability of the absence of similarity issue is that web programming designers may not feel indistinguishable need to settle from numerous deficiencies previously discharge - they can simply be settled later what's more, introduced effectively. Notwithstanding, we have seen no information to demonstrate this is occurring.

7. Time-to-market: Obviously time-to-showcase has dependably been a key business driver, is still vital for web programming. What is bizarre isn't that it is imperative, however that it imparts the spotlight to other quality characteristics. To be sure, being first to showcase is the most vital objective for the majority of the product business. The necessity for persistence can affect the procedure and administration of web programming ventures.

\section{PRINCIPLES}

Web engineering standards and methodologies can acquire the potential disorder Web-based framework improvement under control, limit dangers, and upgrade practicality and quality.

\subsection{Web Engineering and Web Gardening:}

Many Web-based frameworks call for nonstop refresh or refinement, and subsequently Web-based framework improvement may be considered as "persistent, with fine grained advancement, without particular discharges similarly as with programming." In this regard, Web-based framework advancement resembles garden in, like a garden, Web-based framework will proceed to advance, change and develop. Be that as it may, a great introductory framework is required to enable the development to happen in a controlled, however adaptable and predictable way, and to encourage innovativeness, refinement and change. The garden similarity to Webbased framework improvement and the idea of Web as an adaptable medium may make us think, or ponder, for a minute whether Web building approaches are fitting for Web-based framework advancement. We trust that they are fitting, as they are adjusted to Web condition and give adaptability to work inside a structure and permit imaginative advancement. They are not as 'inflexible' as seen by some based on their impression of a portion of the 'customary designing' approaches, and enables inventiveness and personalization to bloom inside a system/constrained limits. Indeed, everything that Web designing promoters is "utilization of sound logical, building and administration standards and restrained and deliberate ways to deal with the fruitful improvement, organization and upkeep of top notch Web-based frameworks and applications." It is suitable if we ensure that the methodologies are suitable to the Web condition. Both the Web designing and Web gardening analogies are legitimate in Web cond ition, and maybe we may need to pursue what is proper from both the methodologies.

The needs for a web site production technology can be obtained by ratifying the root principles of software engineering.

Rigor and formality: A usage innovation ought to give an unmistakable meaning of the substances included in the usage procedure, their connections, and their related semantics. An exact meaning of the attributes and conduct of the diverse components of a Web webpage bolsters designers' comprehension of the current application.

Separation of concerns: A usage innovation should obviously isolate among substance and organizing portrayal, navigational particular, and substance introduction. It ought to be conceivable to characterize distinctive kinds of elements with particular attributes and behavior. Furthermore, the innovation ought to enable the engineer to expand the sort chain of command with application particular composes. An execution innovation ought to offer help for organizing the site substance in an all around characterized way. The ability to characterize navigational examples to get to site assets independently from the displaying of the site's substance ought to be given. The way toward extricating the perspective of the site substance to be conveyed to the end client ought to be indicated independently from the data sources and ought to be parameterized utilizing the entrance setting.

Modularity: A usage innovation ought to give builds and reflections that enable the engineer to partition an intricate issue in littler less complex segments. Modules should utilize data covering up to permit 
International Journal of Research in Advent Technology, Vol.7, No.5, May 2019

\section{E-ISSN: 2321-9637 \\ Available online at www.ijrat.org}

simple coordination and administration. Modularization components could then be utilized to help reuse.

Abstraction: An execution innovation should extract far from low level, immaterial points of interest, recognizing imperative ideas and connections. Moreover, reflection should feature and model ideas that are left certain or covered up in the standard Web innovation.

Anticipation of change: Websites, by their tendency, experience changes continually. It is imperative to give bolster for upkeep. Organizing methods, necessities/outline/execution following, modularization, separation of concerns and deliberations, are the premise of successful Web development.

Generality: A usage innovation ought to give general systems that help the improvement of execution builds and enable the engineer to make specially appointed develops and redo the current ones.

\section{TRENDS}

In perspective of the idea of the Web and Web-based applications, Web building will undoubtedly be a multidisciplinary field, with enveloping contributions from differing territories, for example, human-PC association, UI, frameworks investigation and plan, programming designing, necessities building, hypermedia building, data structures, testing, demonstrating and reproduction and undertaking administration, and in addition sociologies, expressions and realistic outline. In view of the prerequisites, proposition is that an object-oriented modeling structure for the improvement of a World Wide Web website, called WOOM (Web Object Oriented Model). WOOM abstracts from innovative subtle elements and characterizes natives and builds that help adequately the engineer amid the execution and support periods of Web webpage advancement process. The model enables the engineer to characterize the site substance, the navigational structure, and the administration design. A site is actualized by making a WOOM model occurrence, i.e. by instantiating the objects characterized by the model.

The WOOM model case is then naturally converted into ordinary WWW innovations. As indicated by WOOM, a Web webpage can be characterized as far as the accompanying elements: assets, components, locales, servers, connections, and transformers. Assets and components are the principal substances of the model. They model the substance and the structure of an accumulation of data. Assets can be partitioned into compartments and fundamental assets. A holder is an authority of assets. The regulation relationship among assets characterizes a DAG (Direct Acyclic Graph) 1, in which holders are middle of the road hubs and essential assets are clears out. The foundation of the DAG is known as the root holder and encases every one of the assets of the site. Every asset, with the striking exemption of the root holder, is contained at any rate in one compartment. WOOM gives various predefined compartment types: lists, trees, indexes, and sets. Extra holder types can be characterized by the Web originator by broadening the WOOM system. Lists sort out the encased assets in straight design. They are utilized to speak to a consecutive relationship inside a gathering of assets (e.g., the pages that create a guided visit through the books of a given essayist). Trees force a various leveled structure to the encased assets. For instance, the books of a given author can be grouped into classifications: horror, science fiction, Drama, Action and Adventure, etc.; science fiction books, thusly, can be ordered into, say hereditary qualities, space science, and so forth. Indexes sort out the contained assets in two-level trees.

Each container type exports an interface that allows other entities to access the enclosed resources without exposing the container's internal implementation details. For instance, one can address the first resource of a list or the root of a tree without specifying the target resource by name. Basic resources are information repositories. They are distinguished into opaque resources and hyper pages. Opaque resources are unstructured resources. Subclasses of this class are images, i.e., graphic objects, applets, i.e., programs that are activated on the client side, scripts, i.e., applications that are activated on the server side, and external. External resources are those types of information that are not directly supported by the current Web technology and are managed by means of external helper applications. These resources include audio and video information, PostScript files, binaries, and other similar entities. Hyper pages are hypertext pages, which may contain text, anchors, and references to pictures, sounds, and animations. The contents of a hyper page are modeled by a collection of elements .In element is an information unit, like a text paragraph, an anchor, or a dotted list. Elements can be simple or complex. Simple elements are atomic 
International Journal of Research in Advent Technology, Vol.7, No.5, May 2019

\author{
E-ISSN: 2321-9637 \\ Available online at www.ijrat.org
}

data containers, while complex elements contain an ordered list of other elements. For example, the image placeholder element (IMG) is a simple element, while the BODY element may be composed of some paragraphs, a table, etc. Each element belongs atleast to one hyper page. As for resources, the containment relationship among elements defines a DAG. Resources and elements may have some associated attributes. Attributes specify entity properties, such as: expiration date, version, relevance, graphic properties (e.g., the font size of a text string), etc. Each container, hyper page, and complex element associates a unique identifier to each of the enclosed entities. The identifier can be used to denote an entity among others enclosed in the same container, complex element, or hyper page. The identifiers are represented in as labels associated to the containment relationship arcs. The context of a resource (or element) is its position inside the resources/elements containment DAG. Contexts are specified through pathnames. The pathname of an entity is a sequence of identifiers that describes the path from the root container to the entity. This identification mechanism is similar to the wellknown naming scheme based on pathnames adopted by file systems. An entity can be reached through many different paths. As a consequence, an entity can be in different contexts. Each resource and element has an associated translate operation. The invocation of the translate operation for an entity produces its implementation in the conventional WWW technology. That is, hyper pages are translated into HTML files, containers into directories, and elements into character strings representing HTML tags. The translation operation of entities composed by subentities (containers, hyper pages, and complex elements) is recursive. The translation of the composed entity is built using the results of the translation of its sub-entities. For instance, the translation operation of a hyper page invokes the translation operation on the enclosed elements and uses the resulting text strings to build an HTML file.

A site is composed of a root container and one or more servers. A site models a set of related information, represented by the set of resources contained (directly or indirectly) in the site's root container. The associated servers are used to define the network access points to the site contents. A server corresponds, at run-time, to an HTTP daemon process that answers to the end user requests for resources belonging to the site. Each server is characterized by a unique address and has an associated container and context that limit the scope of the resources that are accessible through the server. A WOOM model instance can be translated into a traditional file-based site implementation. The translation process is triggered by calling the translate operation on the root container. The operation propagates in a top-down fashion to the entire WOOM instance DAG. An entity positioned in the DAG in $\mathrm{n}$ different context is translated $\mathrm{n}$ times. This is the case, for example, of a paragraph element enclosed in two different hyper pages. The shared paragraph will correspond, after the translation, to two distinct strings in two different HTML files. This is necessary because conventional WWW technology does not allow information sharing at the element level (two files can not share a piece of text).A predefined set of transformers has been introduced in WOOM to implement navigational patterns that take advantage of the topological structure imposed by some containers (lists, indexes, and trees). For example, a list transformer has been associated to list containers to impose a sequential navigation among the enclosed resources. During the translation process the transformer is able to add proper navigational garnishment to the hyper pages contained in the list. Similarly, a navigational pattern has been predefined for the index container type to allow end users to navigate from the root resource to the leaves and back again. Other transformers have been pre-defined for the tree container in a similar way. Transformers can be used to define properties common to a set of resources, such as color background, graphical decorations, and fonts. Transformers give the opportunity to centralize the control of properties that in conventional WWW technology are defined implicitly on a per-entity basis. Furthermore, transformers are used to extract particular views of a resource or element. During the translation process a transformer can inhibit the translation of portions of information on the basis of their attribute values (e.g., expiration date, creation date, relevance, and version). For instance, let us consider a hyper page containing a scientific paper that is put in three different con- texts. Labeling the page elements with proper attributes, a different view of the contents can be proposed in each context: an abstract, a simplified version without mathematical formalisms, and a detailed presentation. In conclusion, transformers are powerful mechanisms that allow the information provided by a site to be kept separate from the various occurrences in which such information can appear in different forms, within different contexts. By separating the information from its contextual occurrence, we facilitate reuse, 
International Journal of Research in Advent Technology, Vol.7, No.5, May 2019

\section{E-ISSN: 2321-9637 \\ Available online at www.ijrat.org}

implementation, and maintenance of complex data structures. We developed a prototype authoring tool, written in Java, that implements the WOOM model. The tool allows the developer to use WOOM constructs to create the resources that compose a Web site, to perform complex management operations, and to translate the WOOM instance into the standard WWW technology. The main components of the tool are presented in .A first component is the WOOM class framework. The framework provides the definition of basic WOOM entities and provides some predefined constructs. The class framework provides support for representing Web site design elements into the model. This is achieved by means of an integrated, yet separate, design module. The design module is a plug-in component that provides support for a specific design notation. Currently, the HDM notation is supported. As a preliminary step in Web site implementation, the WOOM class framework is imported into the development application. Then, the developer uses the instances of the classes provided by the design module to represent the entities defined during the design phase. Once the design elements have been represented, the developer chooses the corresponding resource implementation. In order to implement each design element, the developer may use the predefined constructs offered by the WOOM class framework or create new application-specific constructs using inheritance, composition, and the transformer mechanism. After suitable constructs have been identified, implementation links that associate a design element with the corresponding implementation construct are created. Such links are used in tracking changes in the implementation to the site design and vice versa. The next step consists of populating the site, by instantiating resource objects of the appropriate classes, and creating application links. Structural links are automatically managed by the semantics of structured objects that implements structured design elements. Once the site has been populated, the translation process is invoked on the site root container. The translation produces the site implementation in terms of files in the file system and servers that provide access to the site's contents. Web site maintenance and management operations are performed on the WOOM model instance.

\section{CONCLUSION}

The WOOM framework offers help for a lot of predefined undertakings like linguistic structure checking, connect refreshing, asset rebuilding, consistency checks, shared asset the board, and configuration change the executives. The model occurrence, after it has been altered, must experience another interpretation procedure so as to think about changes its objective usage. Site cases, made out of site-subordinate schema augmentations (classes and transformers) and asset items are constantly 2 put away in the Repository module. The control application gets to the WOOM schema and occurrences by methods for the WOOM API. The control application is a Java application that uses the natives and administrations offered by the API. We are as of now taking a shot at a graphical interface that enables the designer to get to WOOM benefits in an instinctive and easy to understand way.

\section{REFERENCES}

[1] S. Murugesan, "Web Engineering: A New Discipline for Web-Based System Development", Proc. First Int'l Conf. Software Engineering (ICSE) Workshop on Web Engineering, 1999.

[2] R.S. Pressman, Software Engineering: A Practitioner's Perspective, New York,, 2000.

[3] T.A. Powell, Web Site Engineering: Beyond Web Page Design, Upper Saddle River, N.J.,, 1998.

[4] Web Engineering (Lecture Notes in Computer Science Hot Topics), Heidelberg, Germany, 2001. 